\title{
NEUTRAL ELEMENTS IN GENERAL LATTICES ${ }^{1}$
}

\section{GARRETT BIRKHOFF}

1. Introduction. O. Ore has defined "neutral" elements in modular lattices as elements $a$ satisfying $a \cap(x \cup y)=(a \cap x) \cup(a \cap y)$ for all $x, y$ and dually. ${ }^{2}$ In the case of complemented modular lattices, the neutral elements compose the "center" in J. von Neumann's theories of continuous geometries and regular rings-that is, the set of elements having unique complements. ${ }^{3}$

The purpose of the present note is to extend the notion of neutral elements to general lattices. More precisely, call an element $a$ of a lattice "neutral" if and only if every triple $\{a, x, y\}$ generates a distributive sublattice. It is proved that the neutral elements of any lattice $L$ form a distributive sublattice, consisting of the elements carried into $[I, O]$ under isomorphisms of $L$ with sublattices of direct products. Actually, this sublattice is the intersection of the maximal distributive sublattices of $L$.

Further, complements of neutral elements, when they exist, are unique and neutral. The sublattice of complemented neutral elements may be called the "center" of a lattice: it consists of those elements carried into $[I, O]$ under isomorphisms of $L$ with direct products.

2. Fundamental definition. We define an element $a$ of a lattice $L$ to be "neutral" if and only if every triple $\{a, x, y\}$ generates a distributive sublattice of $L$.

Lemma 1. If a is "neutral," then the dual correspondences $x \rightarrow x \cap a$ and $x \rightarrow x \cup$ a are endomorphisms ${ }^{4}$ of $L$.

Proof. By definition, $(x \cup y) \cap a=(x \cup a) \cap(y \cup a)$ and $(x \cap y) \cap a$ $=(x \cap a) \cap(y \cap a)$, and dually. We note that this condition, which is sufficient to guarantee neutrality in the case of modular lattices, does not guarantee neutrality in general-see the graph below.

1 Presented to the Society, September 8, 1939.

2 O. Ore, On the foundations of abstract algebra I, Annals of Mathematics, (2), vol. 36 (1935), pp. 406-437. For the definitions of lattices and modular lattices (called by Ore structures and Dedekind structures), as well as of sublattice, distributive lattice, $O, I$, and so on, compare the author's Lattices and their applications, this Bulletin, vol. 44 (1938), pp. 793-800-or the author's Lattice Theory, American Mathematical Society Colloquium Publications, vol. 25, 1940.

${ }^{3} \mathrm{~J}$. von Neumann, Lectures on Continuous Geometries, Princeton, 1935-1936. Cf. also R. P. Dilworth, Note on complemented modular lattices, this Bulletin, vol. 45 (1939), pp. 74-76.

4 We define an endomorphism as a homomorphism of $L$ with itself. 
Lemma 2. If $a$ is neutral, then $x \cap a=y \cap a$ and $x \cup a=y \cup$ imply $x=y$.

Proof. By direct computation, using the distributive law twice,

$$
\begin{aligned}
x & =x \cap(x \cup a)=x \cap(y \cup a)=(x \cap y) \mathbf{\cup}(x \cap a) \\
& =(x \cap y) \cup(y \cap a)=y \cap(x \cup a)=y \cap(y \cup a)=y .
\end{aligned}
$$

Using $x$ in the graph, we see that this condition by itself is also not sufficient. However, Lemmas 1-2 together are sufficient to guarantee neutrality.

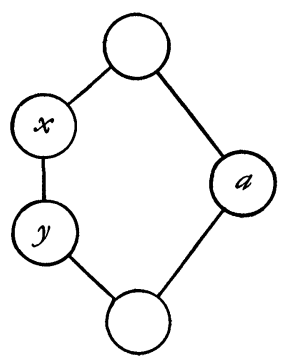

FIG. 1

Indeed, consider the correspondence $x \rightarrow[x \cap a, x \cup a]$ from $L$ to the product ${ }^{5} S T$ of the sublattice $S$ of elements $s \leqq a$, with the sublattice $T$ of elements $t \geqq a$. By Lemma 1 , it is homomorphic onto a sublattice of $S T$; by Lemma 2 , it is one-one; hence it is isomorphic. Moreover $x \rightarrow[a, a]=[I, O]$, since $a$ is the $I$ of $S$ and the $O$ of $T$.

But conversely, $[I, O]$ is obviously "neutral" in $S T$, since each component is. Hence it is neutral in every sublattice of $S T$, including $L$, and we conclude ${ }^{6}$ that the following holds:

Theorem 1. An element of a lattice is neutral if and only if it is carried into $[I, O]$ under an isomorphism of the lattice with a sublattice of a direct product.

3. Neutral elements a sublattice. Just as in the case of modular lattices, we have the following theorem:

THEOREM 2. The neutral elements of any lattice form a distributive sublattice.

Proof. Let $a$ and $b$ be neutral. Then since the product of two

${ }^{5}$ By the "product" $S T$, is meant the system of couples $[s, t], s \varepsilon S, t \varepsilon T$, where $[s, t] \cap\left[s^{\prime}, t^{\prime}\right]=\left[s \cap s^{\prime}, t \cap t^{\prime}\right]$ and dually.

- N.B., we do not assume that $L$ itself has an $O$ or an $I$. 
endomorphisms of $L$ is an endomorphism, it follows that the correspondence

$$
x \rightarrow[x \cap a \cap b,(x \cup a) \cap b,(x \cap a) \cup b, x \cup a \cup b]
$$

defines a homomorphism of $L$ onto a sublattice of a product $S T U V$ of four sublattices of itself. Moreover by Lemma 2, $(x \cap a)$ and $(x \cup a)$ and therefore $x$, are determined uniquely by their images under the homomorphism. Hence the homomorphism is an isomorphism. But $a \cup b$ goes into $[I, I, I, O]$ under this-and hence into $[I, O]$ of $(S T U) V$. Thus $a \cup b$ is neutral; dually, $a \cap b$ is neutral, which was to be proved.

4. Intersection of maximal distributive sublattices. The set of neutral elements of a lattice is also characterized in another way by the next theorem:

THEOREM 3. The set of neutral elements of a lattice $L$ is the intersection of its maximal distributive sublattices.

Proof. First, if $a$ is not neutral, then some triple $\{a, x, y\}$ is not distributive. Hence no maximal distributive sublattice obtained by enlarging the distributive sublattice generated by $\{x, y\}$ can contain $a$. Consequently, the intersection of the maximal distributive sublattices of $L$ contains no non-neutral elements.

Conversely, if $a$ is neutral, and $S$ is a distributive sublattice of $L$, consider the sublattice generated by $\{a, S\}$. The endomorphisms $x \rightarrow x \cap a$ and $x \rightarrow x \cup a$ carry it into sublattices generated by a distributive sublattice and $I$ or $O$. But such sublattices are always distributive-hence so is the sublattice generated by $\{a, S\}$, since it is a sublattice of a product of distributive lattices. Thus every maximal distributive sublattice contains $a$, and the intersection of the maximal distributive sublattices contains every neutral element (as well as no non-neutral elements).

5. Center of a lattice. When one comes to complements of neutral elements, one finds that the following statement holds:

TheOREM 4. Complements of neutral elements, when they exist, are unique and neutral.

Proof. Using Theorem 1 , we see that $[I, O] \mathbf{n}[x, y]$ is $[O, O]$ if and only if $x=O$, while $[I, O] \cup[x, y]$ is $[I, I]$ if and only if $y=I$. Hence $[I, O]$ has no complement except $[O, I]$ in the sublattice of $S T$ isomorphic with $L$, proving uniqueness. Moreover $[O, I]$ is itself neutral, completing the proof. 
COROllary 1. The neutral elements of a complemented lattice form a Boolean algebra.

We define the "center" of a lattice as the set of its complemented neutral elements.

THEOREM 5. The center of any lattice $L$ is a complemented distributive sublattice-and hence a Boolean algebra.

Proof. If $a$ and $b$ are neutral elements of $L$, with (neutral) complements $a^{\prime}$ and $b^{\prime}$, then

$$
\begin{aligned}
& (a \cap b) \cap\left(a^{\prime} \cup b^{\prime}\right)=\left(a \cap b \cap a^{\prime}\right) \cup\left(a \cap b \cap b^{\prime}\right)=O \cup O=O, \\
& (a \cap b) \cup\left(a^{\prime} \cup b^{\prime}\right)=\left(a \cup a^{\prime} \mathbf{u} b^{\prime}\right) \cap\left(b \cup a^{\prime} \mathbf{u} b^{\prime}\right)=I \cap I=I,
\end{aligned}
$$

and so $a \mathbf{n} b$ is complemented. Dually, $a \mathbf{u} b$ is complemented, completing the proof.

We can now specialize Theorem 1 by proving the following:

TheOREm 6. An element is in the center of a lattice $L$ if and only if it is carried into $[I, O]$ under an isomorphism of $L$ with a direct product.

Proof. By Theorem 1, such an element is neutral, and it has the complement $[O, I]$. Conversely, suppose $a$ and $a^{\prime}$ are complementary neutral elements of $L$. Then for all $x$,

$$
x=x \cap I=x \cap\left(a \mathbf{u} a^{\prime}\right)=(x \cap a) \mathbf{u}\left(x \cap a^{\prime}\right) .
$$

Hence the correspondence $x \rightarrow\left[x \cap a, x \cap a^{\prime}\right]$ is one-one between $L$ and the couples $[u, v]$ with $u \leqq a, v \leqq a^{\prime}$; the inverse correspondence is $[u, v] \rightarrow u \cup v$. But it obviously preserves inclusion; hence it is an isomorphism. Finally, it carries $L$ in to the product $S T$ of the lattice $S$ of elements $s \leqq a$, with the lattice $T$ of elements $t \leqq a^{\prime}$, while it carries $a$ into $\left[a \cap a, a \cap a^{\prime}\right]=[I, O]$ in $S T$.

HARVARD UNIVERSITY 